\title{
Patterns of insomnia in depressive states
}

\author{
J. M. HINTON ${ }^{1}$ \\ From the Professorial Unit, Maudsley Hospital, London
}

Sleep is nearly always disturbed in depression and it is commonly believed that the pattern of insomnia is typical or even diagnostic of certain affective disorders. Reactive depression and anxiety are said to cause a delayed onset of sleep and endogenous depression to cause early waking. It is not universally accepted, of course, that reactive and endogenous depressions are separate (Lewis, 1934; Roth, 1960). During trials of hypnotic drugs it was apparent that some patients who demonstrated clinical features in conformity with such depressive syndromes did not show the expected pattern of insomnia.

There are few systematic studies of the sleep of mentally ill patients and these fail to substantiate the belief that neurotic or reactive depression principally delays sleep and endogenous depression cuts it short. Ladame (1923), considering the sleep records of patients, felt unable to distinguish the sleep disturbance of physical from mental disease and even less any of the forms of mental illness. In a study showing the loss of sleep and increased restlessness of depressed patients (Hinton, 1962) it did appear reasonable to differentiate the patients who obtained more of their sleep in the first part of the night from those who slept more in the second part of the night. Those who fell asleep quickly and awoke early had significantly less sleep and were much more restless. The purpose of the present study was to investigate the sleep and motility distribution of depressed patients and see if they were related to features of the depressive state.

\section{METHOD}

Sleep and nocturnal motility have been studied in 34 patients admitted to the Maudsley Hospital with a primary mood change of depression, perhaps admixed with some anxiety. The patients were classified by the medical staff looking after them as $\mathbf{2 7}$ with endogenous and seven with reactive depressions. In some cases this was an easy differentiation, the doctor occasionally using these terms in the diagnosis. In other cases the distinction was dubious but it was usually possible to

1Present address: Academic Department of Psychiatry, Middlesex Hospital, London, W.1. decide from the doctor's written formulation if he considered either the environmental factors or the constitutional factors to be of primary importance. If there was still doubt, the doctor was asked to review the case and decide if the diagnosis was more likely to be acceptable to those who used this classification as endogenous or exogenous. Fortunately for the present purpose it was only necessary to enforce this classification with a few patients and the doctor was not balanced on a knife edge of indecision. A group of 16 patients who had recovered completely from an affective disorder have been similarly observed and compared with the depressed group to confirm that there were sleep changes due to the illness.

During the investigation the patients remained in the same ward and they received no drugs or electroconvulsive therapy (E.C.T.); if hypnotics had been admin-क istered previously a placebo was given on the recorded $\frac{}{\pi}$ nights. The first three nights of recording were used to® enable each patient and the staff to become familiar with the routine and these results were not used. A number of the patients originally selected could not complete the period of study because they recovered rapidly or their condition worsened and they needed early treatment of transfer to another ward. The sleep and motility patterns for each subject were constructed from the first five nights of satisfactory recording after the preliminary period.

The patient's sleep and motility were recorded for the eight hours after his going to bed at 10 p.m. Sleep was recorded by the night nurse, who kept the patient under observation throughout the night and reported on a simple form the times of going to sleep or waking. She also switched on the apparatus for recording motility. This has been modified from the original apparatus (Cox and Marley, 1959; Hinton and Marley, 1959) and the present form has been described elsewhere (Hinton, 1961). Two upright rods were attached to the bed spring under the patient's hips and shoulders. When the patient changed position the rods were displaced and this, in turn, rotated two potentiometers. The potentiometers were connected to an electronic circuit in a side ward, and the voltage changes induced were amplified to operate a pen-recorder. The eight hours of recording each night resulted in 20 feet of paper clearly marking the times of movement. A small movement of the body would score 1 or 2, a major change of position 6 or 8 .

\section{RESULTS}

COMPARISON OF DEPRESSED AND RECOVERED PATIENTS The sleep distribution of each patient has been 
obtained from the five recorded nights by calculating the mean amount of sleep for each of the eight hours, usually 10 p.m. to 6 a.m. To compare the 34 depressed and 16 recovered patients the mean amount of sleep per hour has been determined for each group and shown as histograms in Fig. 1. The raw data on which these are based are not shown (there are 4,000 readings) but the statistical significance of the difference between the two groups has been assessed by analysis of variance. The decrease in the length of sleep from a mean of $7 \cdot 17$ hours in recovered subjects to 5.50 hours in depressed patients was highly significant $(\mathrm{F}=25.6, \mathrm{P}<0.001)$. Any difference in the distribution of sleep could be shown in analysis of variance by calculating the interaction between the groups and the hours, based on the amounts of sleep obtained by the two groups, hour by hour during the night. The distribution of sleep in the depressed group did not differ significantly from that of the recovered group $(F=1.41, P>0.05)$ as depression diminished sleep in each hour of the night.

The mean motility for each hour of the night has been compared between the depressed and recovered patients. In view of the fact that the four beds used for recording could not be calibrated exactly, and different subjects were being compared, it was thought more valid to consider standardized scores rather than raw scores. The total motility score for each night was standardized to 100 , and so the standardized score for each hour was the percentage of the total movement. Although this could not show any alteration in the amount of restlessness it did reveal that there was a significant difference in the distribution of motility $(F=3.03$,
$\mathbf{P}<0.01)$. The recovered patients showed considerable initial movement as they settled in bed (see Fig. 1). Thereafter they moved less, but with a gradual increase throughout the hours of sleep until they began to wake in the morning. This low plateau denoting little movement was not present in the depressed patients, who continued to be restless throughout the night. The increase in total nocturnal motility in depression had been shown in the previous investigation (Hinton, 1962) when the patients were recorded again following their recovery.

COMPARISON OF REACTIVE AND ENDOGENOUS DEPRESsION Having shown the alteration in sleep and motility in the depressed patients, the 34 patients were separated into 27 with endogenous depression and seven with reactive depression. The two groups have been compared in the same manner as the depressed and the recovered patients. The histograms in Fig. 2 show little difference in the sleep pattern, and, likewise, the movement distribution was similar for the two groups. The greater irregularity in the curve of the patients with reactive depression is probably due to the group being smaller and the curves correspondingly less smooth. Using analysis of variance, there was no statistically significant difference in the length of sleep or the distribution of sleep or motility between those described as having endogenous depression and those with reactive depression.

COMPARISON ACCORDING TO FEATURES OF THE ILLNESS The patients' sleep and motility were then subjected to similar group comparisons according to each of 10 selected features of the illness. The
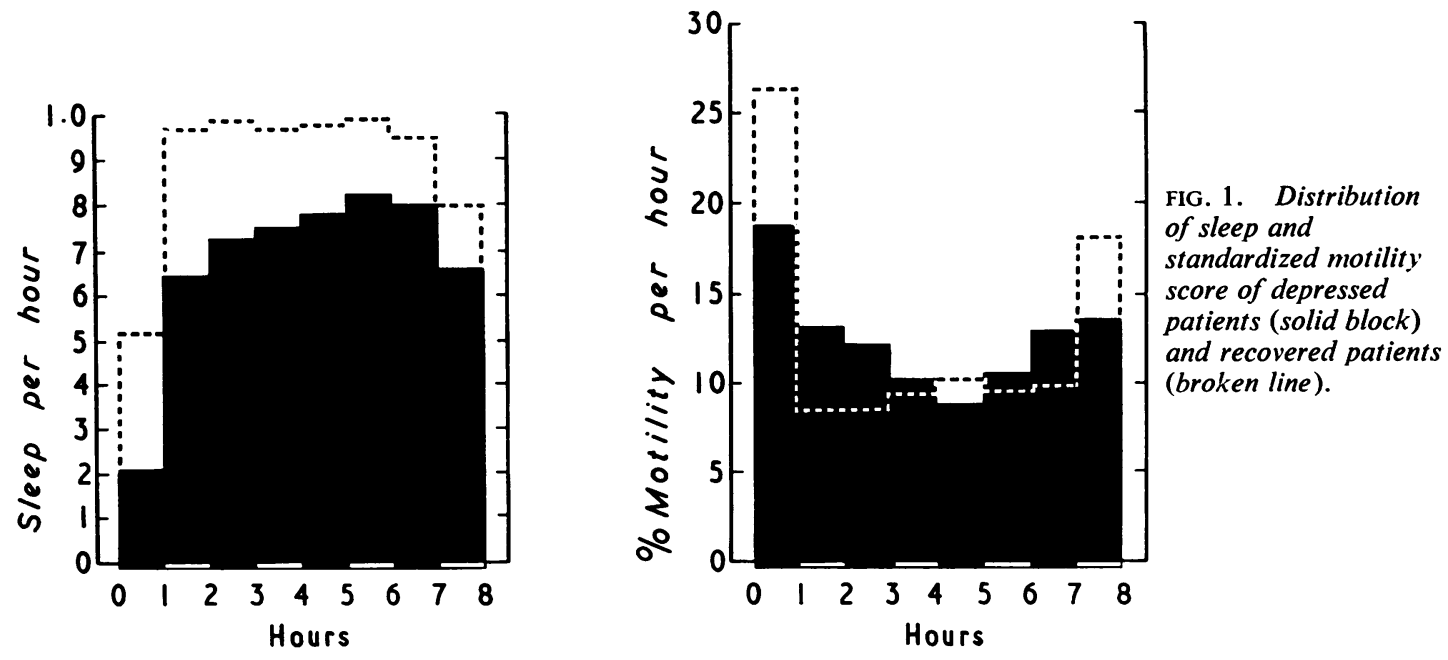


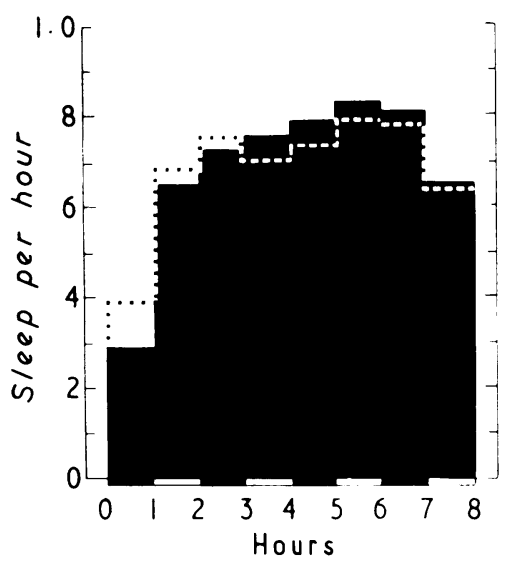

presence or absence of these features was decided by the medical and nursing staff on the day before each night of recording. To help prevent any bias influencing the decisions about clinical features, the analysis of sleep records was not available until the decisions had been made. Six of the 10 factors appeared to make no statistically significant difference to the type of insomnia or distribution of motility. These six factors were, first, a past history of an affective illness sufficient to merit medical attention in 19 patients (there was no difference if the previous illness was restricted to manic-depressive psychosis); second, the illness occurring in the involutional period (taken to be the time of menopausal symptoms or five years after the cessation of menstruation in women, or over the age of 60 in men), occurring in seven of the patients; third, a potential environmental precipitant of the illness operating within three months of its onset, present in 12 patients; fourth, manifest anxiety, present in 21 of the patients; fifth, retardation, with apparent slowing of thought and diminution of activity, present in 13 of the patients, two of whom also showed periods of agitation; and sixth, a substantial improvement with E.C.T., which occurred in 17 of the 22 patients receiving this treatment. It should be noted that two of these groups were very small. Had there been a larger number of involutional patients with the same tendency to lose more sleep in the latter half of the night, this may have become statistically significant. Likewise, the longer sleep obtained by the five patients who showed little change with E.C.T. may have become statistically significant in a larger group.

Three of the considered features of the illness showed a slight association with certain aspects of the sleep disturbance, the level of statistical significance being between $5 \%$ and $10 \%$. First, there was a
FIG. 2. Distribution of sleep and standardized motility score of patients with endogenous depression (solid block) and reactive depression (broken line). trend for those with a family history of affective illness to have a greater reduction of sleep during their depression. Twelve patients had a parent or sib with a history of an affective illness requiring medical advice and the mean sleep per night for this group was 4.98 hours compared with 5.80 hours foro the remainder. Second, the more severely depresse ${ }^{2}$ patients tended to have a greater reduction of sleep $\stackrel{\mathbb{p}}{\oplus}$ in the second half of the night. Thirteen patient usually had a daily assessment of severe depressiong while the remainder were usually assessed as moder ately or mildly depressed. An attempt to use behaviour charts to compare the severity of the il $\vec{E}$ ness between different patients proved to be uno o satisfactory although useful in assessing the progress of a patient. Third, the 16 patients who expressed ideas of being guilty of some fault or that their outlook was hopeless tended to sleep a little longer, 5.67 hours, than the other depressed patients, whose mean length of sleep was $5 \cdot 36$ hours.

These trends did not compare with the highly significant difference between those nine patients who were manifestly agitated and the remaining patients. The agitated patients were very restless, unable to remain in one room, paced the corridors and importuned passing members of the staff, demanding reassurances that they were unable to accept. In one or two the motor component was not so obtrusive but they complained of great mental unrest, fidgeted often, could not settle, and at times drove themselves hard at some physical occupation. Figure 3 shows the greater reduction of sleep in the agitated patients, whose average length of sleep was 4.24 hours compared with 5.97 hours in the remainder $(\mathrm{F}=19.08, \mathrm{P}<0.001)$. The agitated patients lost most sleep in the latter part of the night, a highly significant difference from the other depressed patients $(\mathrm{F}=4.59, \mathrm{P}<0.001)$. Although the dis- 


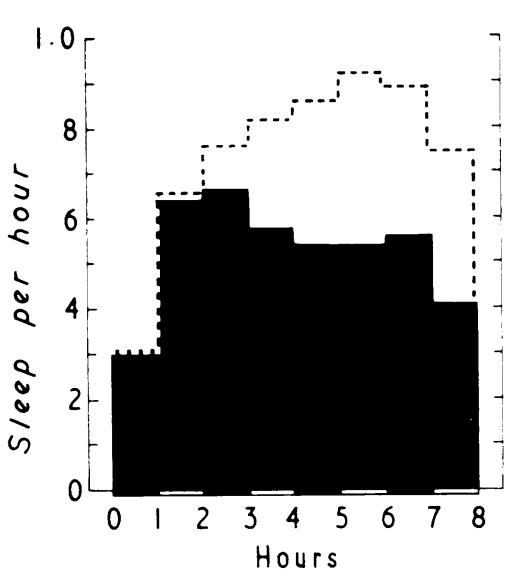

tribution of nocturnal motility appears different on the histogram, it was not statistically significant. The agitated patients had an average motility score of 196 , which contrasted with a mean score of only 86 in the remainder of the group.

\section{DISCUSSION}

The results of this investigation are not in accord with the generally accepted opinions concerning the type of depression and its related pattern of insomnia. It is possible that these beliefs have been built up by selecting evidence favouring preconceived views, but before making this assumption the present study requires criticism and comparison with other investigations. The methods used to assess sleep were imperfect, but although the nurses' observations of sleep and wakefulness and the recording of motility have disadvantages, as discussed elsewhere (Cox and Marley, 1959; Hinton, 1962), they produce measurable results which, in general, agree. Methods of recording the perception of a standard stimulus or measuring the intensity of a stimulus required before a patient responds have been used to assess the level of consciousness but these either require or risk arousing the sleeping subject. Other physiological variables besides motility correlate with sleep (Kleitman, 1944) but, with the exception of the E.E.G. (Loomis, Harvey, and Hobart, 1937), these correlations are inexact (Brooks, Hoffman, Suckling, Kleyntjens, Koenig, Coleman, and Treumann, 1956). Continuous E.E.G. recording for the measurement of sleep has been used (Diaz-Guerrero, Gottlieb, and Knott, 1946; Lester, 1958) but some of the mentally ill patients in this investigation would have found it intolerable for the whole night. There is an association between nocturnal motility and an increase in alpha rhythm in the E.E.G. (Brazier and Beecher, 1952).
The failure to find any significant difference in sleep between patients with reactive depression and those with endogenous depression could be adduced to support the views of those who do not agree that such forms of depression are distinguishable. Those who believe in the distinction might dispute that these patients had been placed in the correct category. When the decisions were taken, however, the medical staff had obtained adequate histories, usually from several sources, and the patient had been observed for some days. The patients had always been discussed between doctors at conferences. If this process failed to separate patients with reactive from those with endogenous depression then it would appear that the criteria of this separation are ambiguous.

It is possible that the selection of patients, especially the rather small group admitted with reactive depression, could have resulted in an atypical sample for investigation. The patients available for inclusion in the study were dependent on the policies of those arranging their admission. It is probable that those admitted would differ from time to time and from hospital to hospital. Patients with a less severe depressive illness are less frequently admitted and Garmany (1958) pointed out that these are more likely to be diagnosed as reactive depression. Another selection was made from these available patients. Some severely ill patients could not be studied sufficiently as the facilities for their treatment were more adequate in other wards. A few patients needed urgent treatment and recording was stopped before sufficient data had been collected. Those patients with an affective disorder, mild or otherwise, who had little or no sleep disturbance were not included in the investigation. The difficulties due to this selection have been in part overcome by making comparisons within the group according to certain aspects of their affective illness. If other 
groups are studied, it is to be anticipated that their selection will be influenced differently. Such groups may be compared with the present one, but it will be more important to see if the presence or absence of various aspects of affective illness considered in this group exert a similar effect upon another group.

The lack of any specific sleep disturbance with reactive or endogenous depression, but rather a finding that both groups have delayed, interrupted, and shortened sleep, was similar to Ladame's findings (1923) in mental disorder. Bauch and Meyer (1955), investigating the occurrence of insomnia in a large number of depressed patients, found it equally frequent in different forms of depression, for example, those with feelings of insufficiency, feelings of sin, agitation, or retardation. Kammerer, Israel, and Geismann (1957), describing their method of recording motility in psychiatric patients, reported that patients with melancholia showed no uniform or characteristic pattern. This contrasted with the opinion of Guttmann (1927) who described marked uniformity in his motility graphs of retarded depressed patients. He found a fairly even distribution of movements throughout the night with only brief periods entirely restful. There was great similarity in the graphs from night to night, seemingly unaltered by drugs. This uniform pattern he differentiated from the records of nervous people ('nervösen') with poor sleep, who showed an irregular distribution and amplitude of movements, varying from night to night, and definitely altered by drugs. It is unfortunate that most of his depressed patients were receiving regular hypnotics during this period of recording, even though Guttmann was convinced that this made no difference to such patients' motility graphs. Kleitman (1939) also found differences between psychoneurotic and depressed patients, but his results contradicted the view that it is the patients with an affective illness who fall asleep more readily and have more disturbed sleep subsequently. The sleep charts kept by nurses and attendants showed that the mean time to fall asleep was 23 minutes for the neurotic and 36 minutes for the affective patients. The interruptions of sleep were more frequent and longer in his psychoneurotic subjects $(0 \cdot 6 /$ night with average duration of $27 \mathrm{~min}$.) than in the depressed subjects $(0.3 /$ night with average duration of $19 \mathrm{~min}$.). The distribution of movement for the first and second halves of the night was 14 and 21 seconds per hour for the psychoneurotic and 19 and 21 seconds per hour for the depressed subjects. There was considerable person-to-person variation, but one cannot deduce from the published results if the difference between the means was statistically significant.

In the present study the absence of a significant difference in the amount of sleep loss in severe as distinct from more moderate affective illnesses was a reflection of the fact that individuals had a wide range of insomnia in response to apparently similar mood changes. There was a trend for the more severely depressed patients to suffer a greater loss of sleep in the second half of the night (between $10 \%$ and $5 \%$ level of probability). This may be related to the fact that five of the 13 severely depressed patients were agitated (see below). Those patients whose depression was characterized by ideas of selfblame, guilt, and punishment, or by lack of hope for the future tended to lose less sleep than the remainder of the group.

Another trend (between $10 \%$ and $5 \%$ level of probability) was the greater loss of sleep amongst the depressed patients who had a parent or sib with a history of affective disorder. There was no difference in the quality of the sleep disturbance, but there exists the possibility that the stronger adverse genetic loading is correlated with greater insomnia in an affective disorder. This finding will need to be repeated at a more significant level statistically before any conclusion can be drawn.

The presence of clinically apparent agitation was $\overrightarrow{+} \overrightarrow{+}$ associated with a greater loss of sleep, especially in $\stackrel{\oplus}{\perp}$ the latter part of the night. There was less than oneo $\frac{}{5}$ in a thousand chance that these differences from the other patients were fortuitous. This group of $\frac{\pi}{0}$ agitated patients closely resembled the others in age and sex distribution. Of the 34 patients with affective $\vec{\bullet}$ disorder, seven had insomnia with a greater loss of sleep in the latter half of the night; five of the seven were agitated. Of the 27 patients whose lack of sleep was more marked in the earlier half of the night, only four were agitated $\left(\chi^{2}=17.75, \mathrm{P}<0.001\right)$. It may be wondered if the alterations in the distribution of sleep and motility in the agitated patients were reflections of the greater loss of sleep. This was contraindicated by comparing them to some other patients who showed an equal sleep loss and finding the sleep distribution was still very different. It appears that one can make an exception to Ladame's usually justified caution in relating mental illness and type of sleep disorder in the case of patients with agitated depression. Such patients are rather more likely to be troubled by early waking than delayed sleep, whereas patients with an affective illness without marked agitation are much more liable to have their greatest loss of sleep in the earlier half of the night.

\section{SUMMARY}

The sleep and nocturnal motility of 34 patients admitted to hospital with an affective illness have been 
investigated. When compared with a group of recovered patients the depressed patients were shown to have reduced sleep in each hour of the night and continued restlessness throughout the night.

There was no significant difference in the insomnia and increased motility between groups classified as 'endogenous' and 'reactive' depressions.

There was no significant relationship between the type of sleep disturbance and a past history of affective illness; illness in the involutional period; environmental precipitant of illness; the presence of anxiety or of retardation; subsequent improvement with electroconvulsive therapy.

There were differing trends (probability between $5 \%$ and $10 \%$ ) in sleep disturbance related to the following factors. Those with a family history of affective illness had a greater reduction in length of sleep; the more severely depressed patients had a greater reduction of sleep in the second half of the night than the less severely depressed; those with ideas of guilt or hopelessness had a lesser reduction in length of sleep than other depressed patients.

The disturbance in sleep of depressed patients with agitation differed very significantly from those without manifest agitation. Agitated patients lost more sleep, most apparent in the latter part of the night.

Some of the possible criticisms of this investigation have been discussed. The results obtained are in closer agreement with the comparatively few published investigations than with current opinions that endogenous depression necessarily causes a patient to wake early and reactive depression delays the onset of sleep.

I wish to thank Professor Sir Aubrey Lewis and Dr. D. L. Davies for their help and encouragement which made the investigation possible, Dr. A. E. Maxwell for his advice on statistics, Mr. G. H. Cox for constructing the apparatus, Miss E. D. Hewland for the illustrations, and members of the nursing staff of the Maudsley Hospital for their careful observations.

\section{REFERENCES}

Bauch, J., and Meyer, H. (1955). Nervenarzt, 26, 121.

Brazier, M. A. B., and Beecher, H. K. (1952). J. appl. Physiol., 4, 819.

Brooks, C. McC., Hoffman, B. F., Suckling, E. E., Kleyntjens, F., Koenig, E. H., Coleman, K. S., and Treumann, H. J. (1956) Ibid., 9, 97

Cox, G. H., and Marley, E. (1959). J. Neurol. Neurosurg. Psychiat., 22, 57.

Diaz-Guerrero, R., Gottlieb, J. S., and Knott, J. R. (1946). Psychosom. Med., 8, 399.

Garmany, G. (1958). Brit. med. J., 2, 341.

Guttmann, E. (1927). Z. ges. Neurol. Psychiat., 111, 309.

Hinton, J. M. (1961). Brit. J. Pharmacol., 16, 82

- (1962). Proc. roy. Soc. Med., 55, 907.

_

Kammerer, T., Israel, L., and Geismann, P. (1957). Cah. Psychiat., 12, 37.

Kleitman, N. (1939). Sleep and Wakefulness. University of Chicago Press, Chicago.

_ (1944). In Medical Physics, edited by O. Glasser, vol. 1, p. 1433. Year Book Publishers, Chicago.

Ladame, C. (1923). Schweiz. Arch. Neurol. Psychiat., 13, 371.

Lester, D. (1958). Science, 127, 1340.

Lewis, A. J. (1934). J. ment. Sci., 80, 277.

Loomis, A. L., Harvey, E. N., and Hobart, G. A. (1937). J. exp. Psychol., 21, 127.

Roth, M. (1960). Comprehens. Psychiat., 1, 135. 